\title{
Erratum to: Constructing ballistic capture orbits in the real Solar System model
}

\author{
Z.-F. Luo - F. Topputo • F. Bernelli-Zazzera • \\ G.-J. Tang
}

Published online: 6 November 2014

C Springer Science+Business Media Dordrecht 2014

\section{Erratum to: Celest Mech Dyn Astr (2014) 120(4) DOI 10.1007/s10569-014-9580-5}

The Figures 8 and 12 of original publication have been revised. The revised figures are given below.

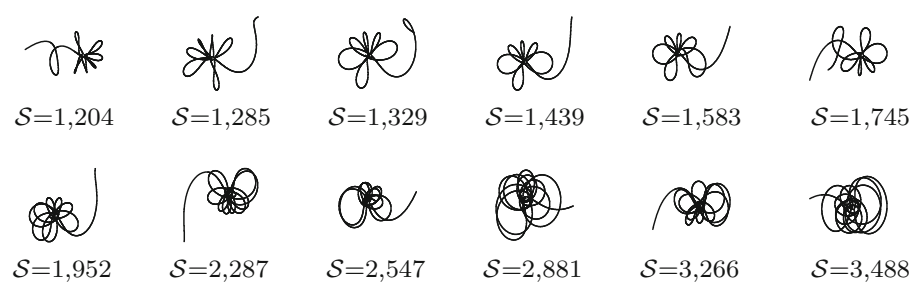

Fig. 8 Ballistic capture orbits about Mercury and their stability index in TU

The online version of the original article can be found under doi:10.1007/s10569-014-9580-5.

Z.-F. Luo · G.-J. Tang

College of Aerospace Science and Engineering, National University of Defense Technology,

Changsha 410073, People's Republic of China

e-mail: luozongfu200@nudt.edu.cn

G.-J. Tang

e-mail: tangguojian@nudt.edu.cn

F. Topputo $(\bowtie) \cdot$ F. Bernelli-Zazzera

Department of Aerospace Science and Technology, Politecnico di Milano, Via La Masa, 34,

20156 Milan, Italy

e-mail: francesco.topputo@polimi.it

F. Bernelli-Zazzera

e-mail: franco.bernelli@polimi.it 


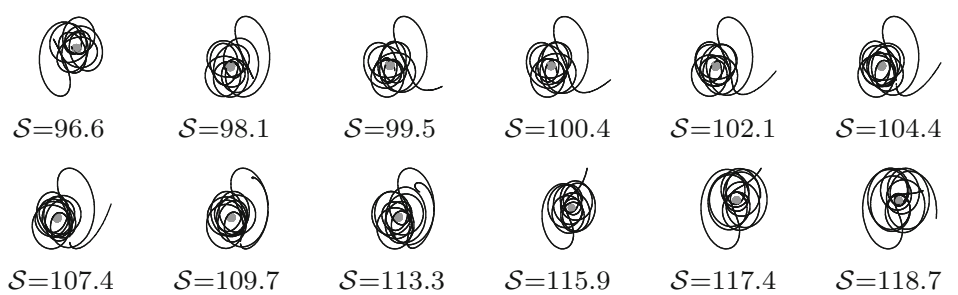

Fig. 12 Ballistic capture orbits about Europa and their stability index in TU 